\title{
Optimal Control of Probability Density Functions of Stochastic Processes *
}

\author{
M. Annunziato ${ }^{1}$ and A. Borzì ${ }^{2}$ \\ ${ }^{1}$ Dipartimento di Matematica e Informatica Università degli Studi di Salerno \\ Via Ponte Don Melillo, 84084 Fisciano (SA), Italia \\ E-mail(corresp.): mannunzi@unisa.it \\ ${ }^{2}$ Università degli Studi del Sannio, Dipartimento e Facoltà di Ingegneria \\ Palazzo Dell'Aquila Bosco Lucarelli, Corso Garibaldi 107, \\ 82100 Benevento, Italia \\ E-mail: alfio.borzi@unisannio.it \\ ${ }^{2}$ Institut für Mathematik und Wissenschaftliches Rechnen, \\ Karl-Franzens-Universität Graz \\ Heinrichstr. 36, 8010 Graz, Austria \\ E-mail: alfio.borzi@uni-graz.at
}

Received April 30, 2010; revised October 1, 2010; published online November 15, 2010

\begin{abstract}
A Fokker-Planck framework for the formulation of an optimal control strategy of stochastic processes is presented. Within this strategy, the control objectives are defined based on the probability density functions of the stochastic processes. The optimal control is obtained as the minimizer of the objective under the constraint given by the Fokker-Planck model. Representative stochastic processes are considered with different control laws and with the purpose of attaining a final target configuration or tracking a desired trajectory. In this latter case, a receding-horizon algorithm over a sequence of time windows is implemented.
\end{abstract}

Keywords: probability density function control, Fokker-Planck equation, optimal control theory, receding-horizon, stochastic process.

AMS Subject Classification: 35Q84; 35K57; 49K20; 60G99; 65C30; 93E20.

\section{Introduction}

Modeling uncertainty is a very active research field with many present and foreseen applications. In this framework, the theory of the stochastic processes [7] provides us established mathematical tools for the description and the analysis of temporal sequences of random quantities in many practical cases. Further,

* Supported in part by the Austrian Science Fund FWF project F3205-N18 "Fast Multigrid Methods for Inverse Problems". 
the possibility to control sequences of events subject to random disturbances is highly desirable for real applications. In this paper, we contribute to the field of control of stochastic processes with the formulation of a framework for the optimal control of the probability density function associated to these processes. We focus on representative continuous time stochastic processes described by the following model

$$
d X_{t}=b\left(X_{t}, t ; u\right) d t+\sigma\left(X_{t}, t\right) d W_{t}
$$

where the state variable $X_{t}$ is subject to deterministic infinitesimal increments of the first addend and to random increments proportional to a Wiener process $d W_{t}$. This is the well-known Itô stochastic differential equation (SDE) [7], where we consider also the action of a time-dependent control $u=u(t)$ that allows to drive the random process to follow a desired trajectory. In deterministic dynamics, the optimal control is achieved by finding the control law $u$ that minimizes a given objective given by a cost functional $J(X, u)$. At least two optimal control design methods are available for this purpose in real applications. These are the model predictive control (MPC) strategy [22] and the linear-quadratic-regulator (LQR) control methodologies [1].

In the present non-deterministic case, the state evolution $X_{t}$ is random and represents an outcome of a probability space, so that a direct insertion of a stochastic process into a deterministic cost functional will result into a random variable. Therefore, when dealing with stochastic optimal control, usually the average of the cost function is considered [4]. In particular, we have

$$
J(X, u)=\mathbb{E}\left[\int_{0}^{T} L\left(t, X_{t}, u(t)\right) d t+\Psi\left[X_{T}\right]\right] .
$$

Notice that this functional is deterministic because of the averaging. This is a Bolza's type cost functional in the finite-horizon case $(T<\infty)$ and it is supposed that the controller knows the state of the system at each instant of time (complete observations). For this case, the method of dynamic programming can be applied $[2,4]$ in order to write the Hamilton-Jacobi-Bellman (HJB) equation for $\inf _{u} J$ with $u$ as optimization function. Some other cases of the cost structure of $J(X, u)$ are quoted in [2], that have application in finance, engineering, and in production planning and forest harvesting. Each $J$ will lead to a different form of the HJB equation that can be analyzed with appropriate methods of partial differential equations [3].

An alternative approach to the HJB formulation for solving the stochastic control problem, consists in approximating the continuous stochastic process by a discrete Markov decision chain [16]. Then, in the framework of dynamic programming, a Bellman equation for this Markov chain is written and solved by using the policy/value iteration. In this approach the information of the controlled stochastic process, carried by the transition probability density function of the approximating Markov process, is utilized to solve the Bellman equation; for details see [20,32].

However, the common methodology to find an optimal controller of random processes consists in reformulating the problem from stochastic to deterministic. This is a reasonable approach when we consider the problem from a 
statistical point of view, with the perspective to find out the collective behavior of the process. In fact, the average $\mathbb{E}[\cdot]$ of the functional of the process $X_{t}$ is omnipresent in almost all stochastic optimal control problems considered in the scientific literature.

The value of the cost functional before averaging is a way to measure the cost of a single process: each realization of $X_{t}$ brings with itself a piece of information of the entire stochastic process modeled by the state equation (1.1). However, the knowledge of the single realization is not useful for the statistical analysis, that would require to determine the average, the variance, and other properties associated to the state of the stochastic process.

On the other hand, the state of a stochastic process can be completely characterized in many cases by the shape of its statistical distribution which is represented by the probability density function (PDF). Therefore, a control methodology defined on the PDF would provide an accurate and flexible control strategy that could accommodate a wide class of objectives. For this reason, in $[5,12,13,31]$ probability density function control schemes were proposed, where the cost functional depends on the PDF of the stochastic state variable. In this way, a deterministic objective results and no average is needed. In $[12,13]$, the objective is defined by the Kullback-Leibler [18] distance between the state PDF and a desired one. On the other hand, in [5, 31] a square distance between the state PDF and a desired PDF is considered.

An important step in our framework, is to recognize that the evolution of the PDF associated to the stochastic process (1.1) is characterized as the solution of the Fokker-Planck (also Fokker-Planck-Kolmogorov) equation; see, e.g., [26, 27]. This is a partial differential equation of parabolic type with Cauchy data given by the initial PDF distribution. Therefore, the formulation of objectives in terms of the PDF and the use of the Fokker-Planck equa tion provide a consistent framework to formulate an optimal control strategy of stochastic processes.

In this paper, our working paradigm for the stochastic optimal control problem is the following. First, we reasonably assume that the PDF of the state variable is known at the initial time. We consider that the state variable $X_{t}$ evolves according to the SDE (1.1) under a controller $u$ which is explicitly modeled in the equation. Then, we consider the Fokker-Planck (FP) equation associated to the process as the governing model. In this way, we have the advantage to be able to characterize the whole shape of the randomness present in the system and avoid the technical difficulties of the stochastic Itô's calculus, since the problem is now formulated in a weak deterministic sense.

We remark that the use of the Fokker-Planck equation for control purposes is a less explored topic. In $[14,15]$, the possibility to control a dynamical system is discussed based on the assumption that the system can be described by a FP equation. In [19], a method that uses a discretized FP equation to solve the HJB equation for the optimal control problem of nonlinear stochastic dynamical systems is presented.

In our framework, we consider a stochastic process in a time interval, with given initial PDF and the objective of approximating a desired final PDF target with the actual PDF of the state variable. Notice that solving the FP 
equation, a time-dependent $\mathrm{PDF}$ is obtained that can describe transient and non-equilibrium statistics. In the time interval, for realistic implementation purposes, we assume that the control is a constant function to be determined by our optimal control scheme once and for all the evolution of the process in the time interval. The cost functional consists of a terminal-time tracking objective and the control cost. The resulting open-loop optimal control problem is formulated as the problem to find a controller that minimizes this cost function within the time interval under the constraint provided by the FP equation.

Further, we use this control strategy applied to a sequence of small time subintervals to construct a fast closed-loop control scheme of the stochastic process based on the receding horizon $(\mathrm{RH})$ model predictive control (MPC) approach $[22,23]$. Notice that in contrast to LQR-type feedback schemes, employed in, e.g., $[5,31]$, the closed-loop schemes based on the RH-MPC approach do not optimize a true performance index; see [8] for a method to quantify the performance degradation. Nevertheless, these schemes provide robust controllers that apply equally well to linear and nonlinear models and allow to accommodate different control- and state constraints [8, 10]. For this reason, RH-MPC schemes are among the most widely used control techniques in process control.

Based on the RH-MPC formulation, we are able to implement a control strategy that allows to determine a piecewise control function that drives the process to follow a desired PDF trajectory, which may include the case of a desired non-equilibrium configuration. In this strategy, measures of the state $\mathrm{PDF}$ are required at the end of each time sub-interval.

In the next section, we define a class of stochastic processes and introduce our framework with optimal control problems based on the FP equation. In Section 3, we discuss the discretization of the FP equation that is required to extend our framework to the case where the FP equation must be solved numerically. Section 4 is devoted to illustrate the receding horizon model predictive control scheme. In Sections 5 and 6, we discuss two basic applications of our formulation of the optimal control problem in the case where the analytic solution of the FP model is known: the Ornstein-Uhlenbeck and the geometric-Brownian processes with additive control. Further, in Section 7 we solve a control problem corresponding to a Shiryaev process. In this case, the closed form solution of the related FP equation is not known, and the numerical scheme of Section 3 is needed. Results of numerical experiments are reported to demonstrate the effectiveness of our FP approach. A section of conclusions completes this work.

\section{Fokker-Planck Optimal Control Formulation of Sto- chastic Processes}

We consider a class of one-dimensional stochastic processes $X_{t} \in \Omega \subset \mathbb{R}$, governed by the following stochastic Itô differential equation

$$
\left\{\begin{array}{l}
d X_{t}=b\left(X_{t}, t ; u\right) d t+\sigma\left(X_{t}, t\right) d W_{t} \\
X_{t_{k}}=X_{k}
\end{array}\right.
$$


where $W_{t}$ is a Wiener process with zero mean and unit variance, $\sigma\left(X_{t}, t\right)>0$ is a function of variance of the stochastic process, $b\left(X_{t}, t ; u\right)$ is a drift term that includes a control function.

Our control strategy is defined in two steps. First, we consider the optimal control for the stochastic model evolving in a given time interval to attain a desired final configuration. We assume that the initial probability density distribution is given and the control is a constant function in this time interval. This is a terminal observation control problem. The second step of our strategy is to consider a sequence of such time intervals $\left(t_{k}, t_{k+1}\right), k=0,1, \ldots, N$, and in each of these intervals we consider a terminal observation control problem where the initial condition for the problem in $\left(t_{k}, t_{k+1}\right)$ is given by the PDF resulting from the controlled evolution of the previous time interval. It is clear that in this way a piecewise control function is obtained while the evolution of the PDF results continuous.

Denote by $f(x, t)$ the probability density to find the process at $x$ at time $t$. Further, let $\hat{f}(x, t ; y, s)$ denotes the transition density probability distribution for the stochastic process to move from $y$ at time $s$ to $x$ at time $t$, which means that $\hat{f}(x, s ; y, s)=\delta(x-y)$, where $\delta$ is the Dirac symbol. Both $f(x, t)$ and $\hat{f}(x, t ; y, s)$ are nonnegative functions and the following holds

$$
\int_{\Omega} f(x, t) d x=1 \quad \text { for all } t \geq s .
$$

This is the conservation condition. The domain $\Omega$ is chosen large enough such that $f(x, t)=0$ for $(x, t)$ on $\Sigma=\Omega \times\left(t_{k}, t_{k+1}\right)$.

If $\rho(y, s)$ is the given initial density probability of the process at time $s$, then we have that the probability density of the process at time $t>s$ is given by the following

$$
f(x, t)=\int_{\Omega} \hat{f}(x, t ; y, s) \rho(y, s) d y .
$$

Notice that $\rho$ should be nonnegative and normalized $\int_{\Omega} \rho(y, s) d y=1$.

Now, we assume to know the initial value of the process at time $t_{k}$, in the sense that we give the probability density $\rho(x, s)$ at time $s=t_{k}$. Our problem is to determine a control $u$ such that starting with initial distribution $\rho$ the process evolves such that a desired target probability density $f_{d}(x, t)$ at time $t=t_{k+1}$ is matched as close as possible. With this setting, we consider the following control problem in $Q=\Omega \times\left(t_{k}, t_{k+1}\right)$. We have

$$
\begin{aligned}
\min J(f, u) & :=\frac{1}{2}\left\|f\left(\cdot, t_{k+1}\right)-f_{d}\left(\cdot, t_{k+1}\right)\right\|_{L^{2}(\Omega)}^{2}+\frac{\nu}{2}|u|^{2}, \\
\partial_{t} \hat{f}\left(x, t ; y, t_{k}\right) & -\frac{1}{2} \partial_{x}^{2}\left(\sigma(x, t)^{2} \hat{f}\left(x, t ; y, t_{k}\right)\right)+\partial_{x}\left(b(x, t ; u) \hat{f}\left(x, t ; y, t_{k}\right)\right)=0, \\
\hat{f}\left(x, t_{k} ; y, t_{k}\right) & =\delta(x-y),
\end{aligned}
$$

where $|u|$ is the absolute value of the (scalar) control and (2.3) is the FokkerPlanck equation [26, 27], i.e. the forward Kolmogorov equation, for the transition density probability distribution $\hat{f}\left(x, t ; y, t_{k}\right)$ of the stochastic process $X_{t}$, 
and $y \in \Omega$. Notice that the solution of the set of equations (2.3)-(2.4) can be interpreted as the fundamental solution of the stochastic process such that for any given $\rho$ the corresponding $f$ is obtained. This problem is as difficult to compute as the Green function of a partial differential equation. However, in our case we assume that the initial distribution $\rho$ is given and hence we can reformulate (2.2)-(2.4) as follows

$$
\begin{aligned}
& \min J(f, u):=\frac{1}{2}\left\|f\left(\cdot, t_{k+1}\right)-f_{d}\left(\cdot, t_{k+1}\right)\right\|_{L^{2}(\Omega)}^{2}+\frac{\nu}{2}|u|^{2}, \\
& \partial_{t} f(x, t)-\frac{1}{2} \partial_{x}^{2}\left(\sigma(x, t)^{2} f(x, t)\right)+\partial_{x}(b(x, t ; u) f(x, t))=0, \\
& f\left(x, t_{k}\right)=\rho(x)
\end{aligned}
$$

where we dropped $s$ in the initial distribution. We consider solutions of the FP equation that are sufficiently regular [6], i.e. $f(x, t)$ has continuous first derivative in time, and continuous second derivative in space, jointly to the conservation condition. However, weaker conditions are possible; see [26].

In the following, we use the optimal control formulation (2.5)-(2.7) to define our control strategy. To illustrate our framework, we consider different stochastic processes and solve (2.5)-(2.7) to obtain the optimal control function.

For completeness, we remark that the optimal control problem (2.5)-(2.7) is an infinite-dimensional constrained minimization problem whose solution is characterized as the solution of the following first-order optimality system:

$\partial_{t} f(x, t)-\frac{1}{2} \partial_{x}^{2}\left(\sigma(x, t)^{2} f(x, t)\right)+\partial_{x}(b(x, t ; u) f(x, t))=0$ in $Q$, (state equation)

$$
f\left(x, t_{k}\right)=\rho(x) \quad \text { in } \Omega, \text { (initial condition) }
$$

$-\partial_{t} p(x, t)-\frac{1}{2} \sigma(x, t)^{2} \partial_{x}^{2} p(x, t)-b(x, t ; u) \partial_{x} p(x, t)=0 \quad$ in $Q$, (adjoint equation)

$p\left(x, t_{k+1}\right)=f_{d}\left(x, t_{k+1}\right)-f\left(x, t_{k+1}\right)$

in $\Omega$, (terminal condition)

$f=0, p=0$

on $\Sigma$, (boundary conditions)

$\nu u+\left(\partial_{x}\left(\frac{\partial b}{\partial u} f\right), p\right)=0$

in $Q$, (optimality equation)

Notice that the state variable evolves forward in time and the adjoint variable evolves backwards in time. In the optimality equation, we have used the following inner product

$$
(\phi, \psi)=\int_{t_{k}}^{t_{k+1}} \int_{\Omega} \phi(x, t) \psi(x, t) d x d t .
$$

The state equation, represented by the FP model (2.6), is a linear partial differential equation of a parabolic type. For a given continuous function $u$, the solution of the FP model is uniquely determined; see, e.g., [11, 26, 27]. 
We denote this dependence by $f=f(u)$ and one can prove that the mapping $u \rightarrow f(u)$ is twice differentiable [21] with respect $x$.

Therefore, we can introduce the so-called reduced cost functional $\hat{J}$ given by $\hat{J}(u)=J(f(u), u)$. The gradient of $\hat{J}$ with respect to $u$ is given by

$$
\nabla \hat{J}(u)=\nu u+\left(\partial_{x}\left(\frac{\partial b}{\partial u} f\right), p\right)
$$

where $p(u)$ is the solution of the adjoint equation for the given $f(u)$.

Summarizing, the optimal control problem (2.5)-(2.7) can be reformulated as solve $\min _{u} \hat{J}(u)$. For a given $u$, the solution of the forward model followed by the solution of the adjoint equation provides the gradient $\nabla \hat{J}(u)$ given by $(2.8)$ that can be used in many optimization schemes [24] to compute the optimal value of the control. However, the use of such optimization schemes becomes necessary in the case where the control $u$ is a vector valued function or it is required to be a function of time. In this paper, we focus on modeling issues and we consider constant control functions such that the solution of $\min _{u} \hat{J}(u)$ can be obtained by implementing the mapping $u \rightarrow f(u)$, i.e. the solution of the state equation, and using a bisection minimization scheme.

\section{Discretization of the Fokker-Planck Equation}

In application, we consider different representative stochastic processes. For some of them the analytic solution of the FP equation is known and can be used to directly solve (2.5)-(2.7). In some other cases, like the Shiryaev process considered below, a numerical solution of the state equation is required. In this section, we discuss the numerical solution of the Fokker-Planck equation by finite differences, when there is no explicit dependence on time of the coefficients $\sigma$ and $b$.

Define a sequence of grids $\left\{\Omega_{h}\right\}_{h>0}$ given by

$$
\Omega_{h}=\left\{x \in \mathbb{R}: x_{j}=j h, j \in \mathbb{Z}\right\} \cap \Omega .
$$

We assume that $\Omega$ is a segment which is a multiple of the spatial mesh size $h$, i.e. $h$ is chosen such that the boundaries of $\Omega$ coincide with grid points. We call $\bar{\Omega}_{h}$ the mesh, $\Omega_{h}$ is the set of interior mesh-points, and $\Gamma_{h}$ is the set of the two boundary end-points.

With $\partial_{x}^{-}$(resp. $\left.\partial_{x}^{+}\right)$denotes the backward (resp. forward) difference quotient in the $x$ direction and a discrete function is extended by 0 on grid points outside of $\Omega$. The second derivative is approximated by the common threepoint stencil and is given by $\Delta_{h}=\partial_{x}^{-} \partial_{x}^{+}$; see [9] for all details.

Let $\delta t$ be the time step size and $N_{t}$ denotes the number of time steps. Define

$$
Q_{h, \delta t}=\left\{\left(x, t_{m}\right): x \in \Omega_{h}, t_{m}=m \delta t, 0 \leq m \leq N_{t}\right\} .
$$

On this grid, $y_{h}^{m}$ denotes a grid function in $\Omega_{h}$ at time level $m$. With $u_{h}$ we denote the optimal control solution of the discrete problem. The action of the forward time difference operator on this function is denoted by

$$
\partial_{t}^{+} y_{h}^{m}=\left(y_{h}^{m+1}-y_{h}^{m}\right) / \delta t
$$


A viable approach to solve (2.6) is to use the mapping $f_{h}^{m}=f_{h}^{m}\left(u_{h}\right)$ defined by the solution of the forward model

$$
\partial_{t}^{+} f_{h}^{m}-\frac{1}{2} \Delta_{h}\left(\sigma_{h}^{2} f_{h}^{m}\right)+\partial_{x}^{C}\left(b_{h}\left(u_{h}\right) f_{h}^{m}\right)=0
$$

with the given initial conditions, and $\sigma_{h}$ and $b_{h}\left(u_{h}\right)$ are the coefficient of the FP equation evaluated on the grid $\Omega_{h}$.

For the discretization of the last term we use a conservative approximation of the spatial derivative $\partial_{x}^{C}[30]$, that fits with the requirement to preserve the conservation of the probability. A conservative scheme is obtained by considering that an approximating polynomial to the flux function $\phi(x):=b(x ; u) f(x, t)$ has the same integral average of the flux in the cell $I_{x}$. Given the numerical flux $\phi_{h}=b_{h}\left(u_{h}\right) f_{h}^{m}$, the reconstruction problem $\Phi$ of the values of $\phi_{h}$ on the boundary of the cell $\hat{\phi}_{j-1 / 2}:=\Phi_{j-1 / 2}\left(\phi_{h}\right)$ and $\hat{\phi}_{j+1 / 2}:=\Phi_{j+1 / 2}\left(\phi_{h}\right)$ is solved. Finally, the difference

$$
\partial_{x}^{C} \phi_{h}:=\left(\hat{\phi}_{j+1 / 2}-\hat{\phi}_{j-1 / 2}\right) / h
$$

gives the conservative approximation to the derivative of the flux to the cell $j$.

Clearly, the term $\partial_{x}^{C}\left(b_{h}\left(u_{h}\right) f_{h}^{m}\right)$ is convective and therefore the direction of the flux has to be considered in order to have a stable discretization. For this purpose, we perform a preventive Lax-Friedrichs flux splitting [30] in order to upwind the derivative in the correct direction. We define the positive and negative fluxes as follows

$$
\phi^{+}(x)=\frac{1}{2}(b(x ; u)+B) f(x, t), \quad \phi^{-}(x)=\frac{1}{2}(b(x ; u)-B) f(x, t),
$$

where $B=\max _{x, u}|b(x ; u)|$. The corresponding numerical approximation to these fluxes ia given as follows

$$
\phi_{h}^{+}=\frac{1}{2}\left(b_{h}\left(u_{h}\right)+B\right) f_{h}^{m}, \quad \phi_{h}^{-}=\frac{1}{2}\left(b_{h}\left(u_{h}\right)-B\right) f_{h}^{m} .
$$

For the reconstruction problem, we have taken three cells. Consider the stencil $\{j-1, j, j+1\}$ with reconstruction coefficients $c^{+}=[-1 / 6,5 / 6,1 / 3]$ for the positive flux, so that at the right boundary of the cell $j$, we have

$$
\hat{\phi}_{j+1 / 2}^{+}=-1 / 6 \phi_{j-1}^{+}+5 / 6 \phi_{j}^{+}+1 / 3 \phi_{j+1}^{+} \text {. }
$$

Similarly, for the negative reconstructed flux $\hat{\phi}_{j+1 / 2}^{-}$we use the stencil $\{j, j+$ $1, j+2\}$ with coefficients $c^{-}=[1 / 3,5 / 6,-1 / 6]$. Therefore the total flux for the right boundary of the cell $j$ is given by

$$
\hat{\phi}_{j+1 / 2}=\hat{\phi}_{j+1 / 2}^{+}+\hat{\phi}_{j+1 / 2}^{-} .
$$

The same reconstruction is performed for the cell $j-1$ and $\partial_{x}^{C}$ is calculated.

In order to ensure convergence of the numerical scheme, the following CourantFriedrichs-Lewy condition $\delta t=c \min \left(h^{2} /\left(2\left\|\sigma_{h}^{2}\right\|_{\infty}\right), h / B\right)$ must be satisfied. We find that $c=0.8$ guarantees stable and accurate numerical results. 


\section{A Receding-Horizon Model Predictive Control Scheme}

Our purpose is to control the probability density function of a stochastic process to track a given sequence of desired PDFs in time. Let $(0, T)$ be the time interval where the process is considered. We assume time windows of size $\Delta t=T / N$ with $N$ a positive integer. Let $t_{k}=k \Delta t, k=0,1, \ldots, N$. At time $t_{0}$, we have a given initial PDF denoted with $\rho$ and with $f_{d}\left(\cdot, t_{k}\right), k=1, \ldots, N$, we denote the sequence of desired PDFs. Our scheme starts at time $t_{0}$ and solves the minimization problem $\min _{u} J(f(u), u)$ defined in the interval $\left(t_{0}, t_{1}\right)$. Then, with the probability density function $f$ resulting at $t=t_{1}$ solving the optimal control problem, we define the initial PDF for the subsequent optimization problem defined in the interval $\left(t_{1}, t_{2}\right)$. This procedure is repeated by receding the time horizon until the last time window is reached. This is an instance of the class of receding horizon model predictive control (RH-MPC) schemes $[23,22]$ that is widely used in engineering applications to design closed-loop algorithms. One important aspect of this approach is that it can be applied to infinite dimensional evolution systems [10], that is the case of the FP model.

The RH-MPC procedure is summarized in the following algorithm.

Algorithm 1 [RH-MPC Control]. Set $k=0$; assign the initial PDF, $f\left(x, t_{k}\right)=$ $\rho(x)$ and the targets $f_{d}\left(\cdot, t_{k}\right), k=0, \ldots, N-1$;

1. In $\left(t_{k}, t_{k+1}\right)$, solve $\min _{u} J(f(u), u)$ (bisection).

2. With the optimal solution $u$ compute $f\left(\cdot, t_{k+1}\right)$.

3. Assign this PDF as the initial condition for the FP problem in the next time window.

4. If $t_{k+1}<T$, set $k:=k+1$, go to step 1 and repeat.

5. End.

In the following, we report results of numerical experiments where we compute the control $u$ that solves control problems corresponding to different stochastic processes. In the numerical RH-MPC procedure, a quadrature is required for the computation of the norm in (2.5); for this purpose we use a trapezoidal formula.

In the case of the Ornstein-Uhlenbeck process and the geometric-Brownian process, we have the analytical solution of the Fokker-Planck equation (2.3) in closed form. Inserting this solution in (2.1) and using numerical quadrature, we are able to compute the PDF without solving the FP equation numerically. We use this fact, to validate our RH-MPC algorithm, while all results presented in the following sections are obtained by solving the FP equation with our numerical scheme. In the cases where the analytical solution is known, we obtain optimal controls that approximate very well optimal controls computed using the exact FP solution. 


\section{An Ornstein-Uhlenbeck Process with Additive Control}

The classical problem of a massive particle immersed in a viscous fluid and subject to random Brownian fluctuations due to interaction with other particles, is modeled by the Ornstein-Uhlenbeck (OU) process. We focus on the control of the PDF of the OU process. For this purpose, we set $b\left(X_{t}, t ; u\right)=-\gamma X_{t}+u$, $\sigma\left(X_{t}, t\right)=\sigma$, where $X_{t}$ represents the velocity of the particle and $u$ is the momentum induced by an external force field defining the control mechanism. With this setting, the Fokker-Planck equation for transition probabilities under the action of the control $u$ becomes

$$
\begin{aligned}
& \partial_{t} \hat{f}\left(x, t ; y, t_{k}\right)-\frac{1}{2} \sigma^{2} \partial_{x}^{2} \hat{f}\left(x, t ; y, t_{k}\right)+\partial_{x}\left((u-\gamma x) \hat{f}\left(x, t ; y, t_{k}\right)\right)=0, \\
& \hat{f}\left(x, t_{k} ; y, t_{k}\right)=\delta(x-y) .
\end{aligned}
$$

In this case, the solution to the Fokker-Planck equation is well known to be a Gaussian distribution with mean

$$
\mu\left(t ; y, t_{k} ; u\right)=u / \gamma+(y-u / \gamma) e^{-\gamma\left(t-t_{k}\right)}
$$

and variance

$$
\bar{\sigma}^{2}\left(t, t_{k}\right)=\frac{\sigma^{2}}{2 \gamma}\left(1-e^{-2 \gamma\left(t-t_{k}\right)}\right) .
$$

Therefore the solution is as follows

$$
\hat{f}\left(x, t ; y, t_{k} ; u\right)=\frac{1}{\sqrt{2 \pi \bar{\sigma}^{2}\left(t, t_{k}\right)}} \exp \left(-\frac{\left(x-\mu\left(t ; y, t_{k} ; u\right)\right)^{2}}{2 \bar{\sigma}^{2}\left(t, t_{k}\right)}\right) .
$$

This solution defines a mapping $\hat{f}=\hat{f}(u)$. Now, assuming an initial distribution $\rho$ at $t=t_{k}$, and having $\hat{f}\left(x, t_{k+1} ; y, t_{k} ; u\right)$, the final distribution $f\left(x, t_{k+1} ; u\right)$ is given by integration as defined in (2.1). This procedure provides a mapping $f=f(u)$ and thus the following reduced cost functional is obtained

$$
J(f(u), u)=\frac{1}{2}\left\|(f(u))\left(\cdot, t_{k+1}\right)-f_{d}\left(\cdot, t_{k+1}\right)\right\|_{L^{2}(\Omega)}^{2}+\frac{\nu}{2}|u|^{2} .
$$

We obtain the one-parameter optimization problem $\min _{u} J(f(u), u)$. This problem is solved efficiently by a bisection minimization procedure [24].

In Figure 1, we show the optimal solution for the problem (5.1) corresponding to the Ornstein-Uhlenbeck process. These results are obtained by solving (2.6) with our numerical scheme and appear to be very accurate compared to the results obtained using (5.2) and (2.1).

In this experiment, the parameters are $\gamma=1, \sigma=0.8, \nu=0.1$. The initial distribution (see Figure 1, black dotted line) is a Gaussian with zero mean and variance $\sigma=0.1$. The target is also Gaussian as follows

$$
f_{d}(x, t)=\frac{1}{\sqrt{2 \pi \sigma^{2}}} \exp \left(-\frac{(x-v(t))^{2}}{2 \sigma^{2}}\right)
$$


with mean value following the law $v(t)=2 \sin (\pi t / 5)$ and variance $\sigma=0.2$, for $t=t_{1}, \ldots, t_{N}-1$, where the optimal distribution (see Figure 1, solid line) is calculated at steps of $\Delta t=0.5$ until time $T=5$, with $u$ in the set $u \in[-4,4]$. At the right-hand side of Figure 1 the time evolution of the control $u$ is shown. The spatial mesh size is $h=0.05$ and $\delta t=1.6 \cdot 10^{-3}$.
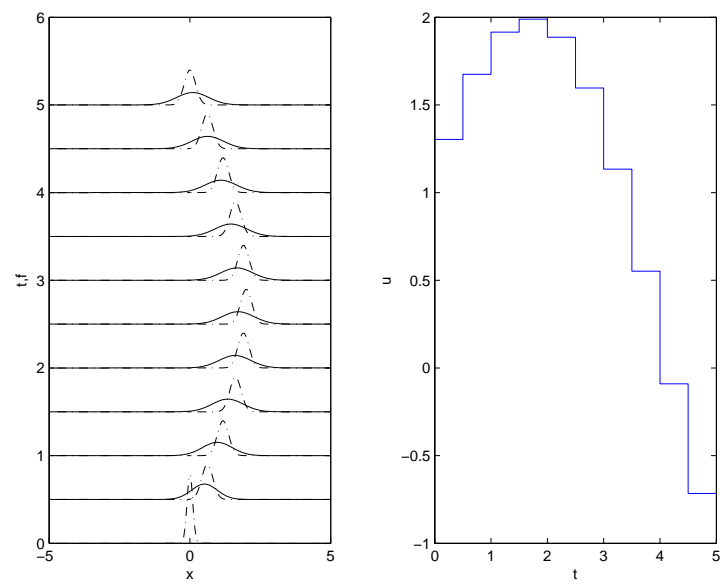

Figure 1. The optimal control of the Ornstein-Uhlenbeck process. Left-hand side: computed PDF (solid line) and desired PDF (dotted-dashed line) at different time windows. Right-hand side: The optimal control function.

\section{A Geometric-Brownian Process with Additive Drift Control}

The Geometric-Brownian motion is the most widely used stochastic process in financial modeling; see, e.g., [17]. The classical Merton's portfolio problem models the wealth as an Itô stochastic process that generates a GeometricalBrownian (GB) motion. Further, its properties are building blocks for modeling a wide variety of exotic options and other derivative contracts. In this case, control may be required to drive a portfolio to a given value.

In the GB control problem, we have $b\left(X_{t}, t ; u\right)=(\mu+u) X_{t}, \sigma\left(X_{t}, t\right)=\sigma X_{t}$. As an example of application, we assume that $X_{t}$ is the wealth, $\mu$ is the average market price of return including the fraction of the portfolio invested in risky market, $\sigma$ is the volatility parameter. In this model, we insert the controller $u$ as a fraction of the portfolio invested in risk free and constant interest rate market, such as a government bond. The following Fokker-Planck equation results

$$
\begin{aligned}
& \partial_{t} \hat{f}\left(x, t ; y, t_{k}\right)-\frac{1}{2} \sigma^{2} \partial_{x}^{2}\left(x^{2} \hat{f}\left(x, t ; y, t_{k}\right)\right)+(\mu+u) \partial_{x}\left(x \hat{f}\left(x, t ; y, t_{k}\right)\right)=0 \\
& \hat{f}\left(x, t_{k} ; y, t_{k}\right)=\delta(x-y)
\end{aligned}
$$

where $x, y \geq 0$. In this case, the analytical solution for the transition probability 
function is known as the following log-normal distribution

$$
\hat{f}\left(x, t ; y, t_{0} ; u\right)=\frac{1}{x \sqrt{2 \pi \sigma^{2}\left(t-t_{0}\right)}} \exp \left(-\frac{\left[\log (x / y)-\left(\mu+u-\sigma^{2} / 2\right)\left(t-t_{0}\right)\right]^{2}}{2 \sigma^{2}\left(t-t_{0}\right)}\right) .
$$

Proceeding as in the previous OU case, we obtain an one-parameter optimization problem that can be solved efficiently by bisection.
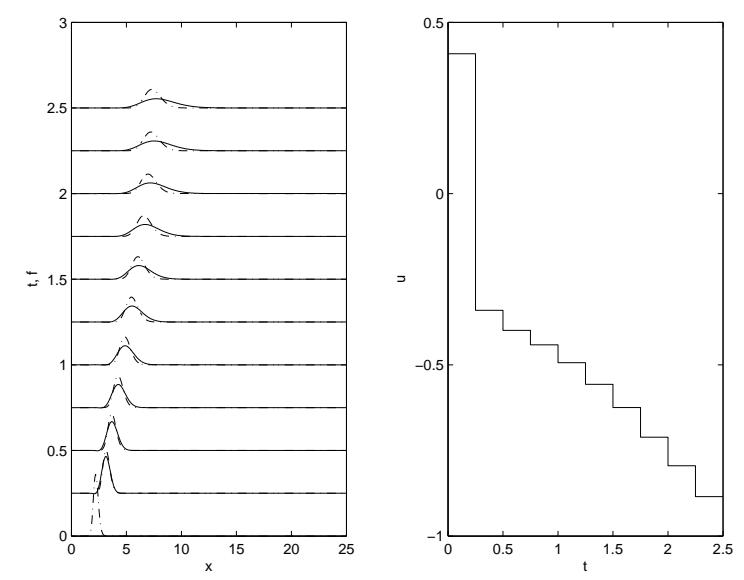

Figure 2. The optimal control of the geometric Gauss process. Left-hand side: computed PDF (solid line) and desired PDF (dotted-dashed line) at different time windows. Right-hand side: The optimal control function.

In Figure 2, we show the results obtained with the Geometric-Brownian process. We compute the numerical solution to the FP equation. In this experiment, the parameters are $\mu=1, \sigma=0.1$ and $\nu=0.1$. The initial and target distributions are in the following log-normal form

$$
f_{d}(x, t)=\frac{1}{x \sqrt{2 \pi \sigma^{2}}} \exp \left(-\frac{[\log (x)-\tilde{\mu}(t)]^{2}}{2 \sigma^{2}}\right) .
$$

Specifically, the initial distribution (see Figure 2, dotted-dashed line) $\rho(x)=$ $f_{d}\left(x, t_{0}\right)$ is log-normal with $\tilde{\mu}\left(t_{0}\right)=0.8, \sigma=0.1$. The target distribution (see Figure 2, solid line) $f_{d}(x, t)$ is $\log$-normal with $\tilde{\mu}(t)=1+\sin (\pi t / 5)$ and $\sigma=0.1$ for $t=t_{1}, \ldots, t_{N}-1$, where the optimal distribution is calculated at time steps $\Delta t=0.25$, until the time $T=2.5$. The step size for space is $h=0.0625$, and time $\delta t=2.4752 \cdot 10^{-4}$. The search set for the control is $u \in[-1,0.5]$. The plot at the right-hand side of Figure 2 shows the time evolution of the control.

\section{The Shiryaev Process}

An interesting setting in application is $b\left(X_{t}, t ; u\right)=u+\mu X_{t}, \sigma\left(X_{t}, t\right)=\sigma X_{t}$. In this case, the control is not multiplying $X_{t}$ in the drift term. This model is known in the literature as the Shiryaev process [25] and it can be found in applications in sequential analysis and financial mathematics $[25,28,29]$. This 
setting can be considered as the sum of absolute fixed income from risk-free market and outcome of consumption. The Fokker-Planck equation corresponding to the Shiryaev process is as follows

$$
\begin{aligned}
& \partial_{t} \hat{f}\left(x, t ; y, t_{k}\right)-\frac{1}{2} \sigma^{2} \partial_{x}^{2}\left(x^{2} \hat{f}\left(x, t ; y, t_{k}\right)\right)+\partial_{x}\left((u+\mu x) \hat{f}\left(x, t ; y, t_{k}\right)\right)=0, \\
& \hat{f}\left(x, t_{k} ; y, t_{k}\right)=\delta(x-y) .
\end{aligned}
$$

Although close to the GB case (6.1), the analytical solution to the FP equation is not known in closed form; however some series expansion can be obtained [25]. Therefore, we solve the following FP equation numerically. We have

$$
\partial_{t} f(x, t)-\frac{1}{2} \sigma^{2} \partial_{x}^{2}\left(x^{2} f(x, t)\right)+\partial_{x}((u+\mu x) f(x, t))=0 .
$$
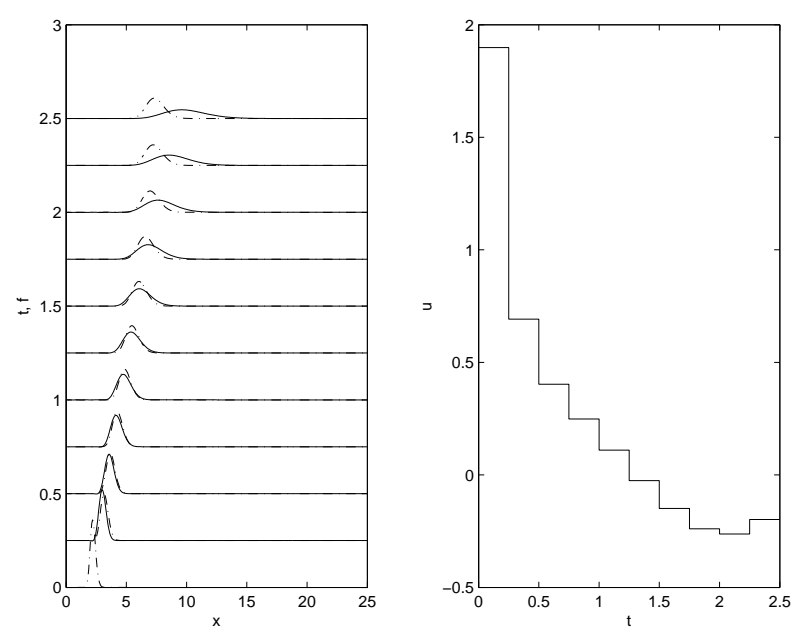

Figure 3. Optimal control of the Shiryaev process. Left-hand side: computed PDF (solid line) and desired PDF (dotted-dashed line) at different time windows. Right-hand side: optimal control function.

In Figure 3, we plot results of our calculation with $\sigma=0.1, \mu=0.5$, spatial mesh $h=0.0625$ and time $\delta t=2.4752 \cdot 10^{-4}$ size, $u \in[-1,3]$. Initial and desired PDF functions are chosen as in the Geometric-Brownian process case; see (6.2). The plot at the right-hand side of Figure 3 shows the time evolution of the control.

\section{Concluding Remarks}

In this paper, a Fokker-Planck framework for determining controls of stochastic processes was presented. The control objective was defined based on the probability density function. The control strategy is based on a receding-horizon model predictive control framework where optimal controls were obtained minimizing the objective under the constraint given by the Fokker-Planck equation 
that models the evolution of the probability density function. Representative stochastic processes with different control laws were considered. Results of numerical experiments demonstrated the effectiveness of the proposed approach.

\section{References}

[1] D. Bertsekas. Dynamic Programming and Optimal Control, Vols. I and II. Athena Scientific, 2007.

[2] V.S. Borkar. Controlled diffusion processes. Probab. Surv., 2:213-244 (electronic), 2005. ISSN 1549-5787. Doi:10.1214/154957805100000131.

[3] M. Falcone. Appendix to the book optimal control and viscosity solutions of Hamilton-Jacobi-Bellman equations. In M. Bardi and I. Capuzzo Dolcetta(Eds.), Numerical Solution of Dynamic Programming Equations. Birkhauser, Boston, 2008 .

[4] W. H. Fleming and R. W. Rishel. Deterministic and Stochastic Optimal Control. Applications of Mathematics. Springer-Verlag, Berlin, 1975.

[5] M. G. Forbes, M. Guay and J. F. Forbes. Control design for first-order processes: shaping the probability density of the process state. Journal of process control, 14:399-410, 2004. Doi:10.1016/j.jprocont.2003.07.002.

[6] A. Friedman. Partial Differential Equations of Parabolic Type. Dover Publications, 2008.

[7] Ц̆. I. GThman and A.V. Skorohod. Stochastic differential equations. SpringerVerlag, New York, 1972. Translated from the Russian by Kenneth Wickwire, Ergebnisse der Mathematik und ihrer Grenzgebiete, Band 72

[8] L. Grüne and A. Rantzer. On the infinite horizon performance of receding horizon controllers. IEEE Trans. Aut. Control, 53:2100-2111, 2008.

Doi:10.1109/TAC.2008.927799.

[9] W. Hackbusch. Elliptic Differential Equations. Springer-Verlag, New-York, 1962.

[10] K. Ito and K. Kunisch. Receding horizon optimal control for infinite dimensional systems. ESAIM, 35:814-824, 1990.

[11] R. Jordan, D. Kinderlehrer and F. Otto. Variational formulation of the FokkerPlanck equation. SIAM J. Math. Anal., 29:1-17, 1998.

Doi:10.1137/S0036141096303359.

[12] G. Jumarie. Tracking control of nonlinear stochastic systems by using path crossentropy and Fokker-Planck equation. Internat. J. Systems Sci., 23(7):1101-1114, 1992. ISSN 0020-7721. Doi:10.1080/00207729208949368.

[13] M. Kárný. Towards fully probabilistic control design. Automatica, 32(12):17191722, 1996. Doi:10.1016/S0005-1098(96)80009-4.

[14] A.A. Krasovskiy. The Fokker-Planck-Kolmogorov equation and the synthesis of linear control systems. II. Engrg. Cybernetics, 1968(2):1-6, 1968. ISSN 0013$788 \mathrm{x}$.

[15] A.A. Krasovskiy. The Fokker-Planck-Kolmogorov equation and the synthesis of relay control systems. Engrg. Cybernetics, 1967(5):45-53, 1968. ISSN 0013$788 \mathrm{x}$. 
[16] J. Krawczyk. A Markovian approximated solution to a portfolio management problem. ITEM. Inf. Technol. Econ. Manag., 1(1):Paper 2, 33 pp. (electronic), 2001. ISSN 1643-8949.

[17] J. Krawczyk. On loss-avoiding payoff distribution in a dynamic portfolio management problem. The Journal of Risk Finance, 9(2):151-172, 2008. Doi:10.1108/15265940810853913.

[18] S. Kullback. Information theory and statistics. John Wiley and Sons, Inc., New York, 1959.

[19] M. Kumar, S. Chakravorty and J.L. Junkins. Computational nonlinear stochastic control based on the Fokker-Planck-Kolmogorov equation. AIAA Guidance, Navigation and Control Conference and Exhibit, Honolulu, Hawaii, Aug. 18-21, 2008, pp. AIAA-2008-6477, 2008.

[20] H.J. Kushner. Numerical methods for stochastic control problems in continuous time. SIAM J. Control Optim., 28(5):999-1048, 1990. ISSN 0363-0129. Doi:10.1137/0328056.

[21] J. L. Lions. Optimal Control of Systems Governed by Partial Differential Equations. Springer, Berlin, 1971.

[22] L. Magni, D.M. Raimondo and F. Allgöwer. Nonlinear Model Predictive Control. Springer, Berlin, 2009. Doi:10.1007/978-3-642-01094-1.

[23] D. Q. Mayne and H. Michalska. Receding horizon control for nonlinear systems. IEEE Trans. Aut. Control, 35:814-824, 1990. Doi:10.1109/9.57020.

[24] J. Nocedal and S.J. Wright. Numerical Optimization. Springer-Verlag, 2006.

[25] G. Peskir. On the fundamental solution of the Kolmogorov-Shiryaev equation. In Yu. Kabanov, R. Liptser and J. Stoyanov(Eds.), From Stochastic Calculus to Mathematical Finance, pp. 535-546. Springer, Berlin, 2006. Doi:10.1007/978-3-540-30788-4-26.

[26] S. Primak, V. Kontorovich and V. Lyandres. Stochastic Methods and Their Applications to Communications. John Wiley \& Sons, Chichester, 2004. Doi:10.1002/0470021187.

[27] R. Risken. The Fokker--Planck Equation: Methods of Solution and Applications. Springer, Berlin, 1996.

[28] A. N. Shiryaev. The problem of the most rapid detection of a disturbance in a stationaty process. Soviet Mathematics, 2(2):795-799, 1961.

[29] A. N. Shiryaev. Quickest detection problems in the technical analysis of the financial data. In H. Geman, D. Madan, S.R. Pliska and T. Vorst(Eds.), Math. Finance Bachelier Congress (Paris 2000), pp. 487-521. Springer, 2002.

[30] Ch.W. Shu. Essentially non-oscillatory and weighted essentially non-oscillatory schemes for hyperbolic conservation laws. In A. Quarteroni(Ed.), Advanced Numerical Approximation of Nonlinear Hyperbolic Equations. Lecture Notes in Mathematics, volume 1697, pp. 325-432. Springer, 1998.

[31] H. Wang. Robust Control of the Output Probability Density Functions for Multivariable Stochastic Systems with Guaranteed Stability. IEEE Trans. on Automatic Control, 44(11):2103-2107, 1999. Doi:10.1109/9.802925.

[32] L. Wang and F. Bai. The state equations methods for stochastic control problems. Numer. Math. Theor. Meth. Appl., 1(3):79-96, 2010. 